\title{
Clinical prolonged follow up of patients with advanced cancers after chemotherapy with traditional medicine $(78$ cases)
}

\author{
George Zhu* \\ The Institute of Oncology, Tehran University of Medical Sciences, Tehran
}

\begin{abstract}
Objective: Traditional systems of medicine all over the world even traditional medicine and cancer have been using plants and plants products for therapeutic intention. The purpose of this retrospective trials is to assesses the clinical efficacy of chemotherapy in conjunction with TCM for a broad variety of cancers.

Methods: 78 patients with available cancers were concluded in the study during September1993 - May2018. The sex ratio of male: female was 53:25 respectively. The mean age at onset was 46.9 years (range 10-79 years). All patients were treated with different dosage of various chemotherapy in combination with TCM or traditional medicine alone. The basic chemotherapeutic regimen consisted of vincristine (VCR,1-2mg/week), cyclophosphamide (CTX, 200-1,000mg / week), mitomycin C (MMC, 2-4mg/week) and 5-fluorouracil (5-Fu,250-500mg/day). In addition, according to patient condition, the additional drug doxorubicin (ADM,20mg/week) in lymphoma and metastatic breast cancer, demethylcantharidin in liver cancer and cisplatin (DDP) or interleukin-2(PHA) or gefitinib in lung cancer. The detail prescription of TCM varied among a broad variety of carcinomas (see full text case reports). The criteria of complete remission (CR) and/or partial remission (PR)is according to the rules where physicians have in common with in clinics.

Results: In 78 cases, the CR was obtained in 33(42.3\%) advanced cancers, a short CR in $12(15.4 \%)$ cases, PR in 27 (34.6\%) cancers, Stable disease in 6 cases. In differential types of 20 patients over ten years, lymphoma occupied 8 cases (40\%). As to approach to the schedule of drug administration,16 lymphoma obtained CR via COMA (CTX, VCR, MMC, ADM) regimen and TCM or antibiotics and immunotherapy. Five advanced gastric cancer were successfully treated using MFC and cinobufacini/cantharidin, and TCM. In follow up, one HCC accompanied with colon polyps obtained CR via hepatectomy and targeting oncogenic receptor tyrosine kinase inhibitor sorafenib. Among two lung cancer, one female with metastatic lung cancer was given targeting oncogenic receptor EGFR gefitinib therapy after the combination chemotherapy, which was stable disease for $8+$ months.CR can also be achieved in one advanced cholangicarcinoma and one advanced gallbladder cancer through major protocol of TCM and the addition of small dosage of chemotherapy. Thyroid cancer was placed on the primary use of TCM. The crude herbs consisted of sargassum, tangle, Oyster (mussels), Poria cocos, Ophiopogon japonicus, Prunella vulgaris, Taraxacum, Scrophularia ningpoensis, Cremastra appendiculata, Trichosanthes Kirilowii, Sophora subprostrata, Houttuynia cordata, Scutellaria barbata d. don and Oldenlandia diffusa roxb. Among those long-term survivors, 31 carcinomas obtained in disease-free survival over 5years, 20 cancers were survival over 10years, the longest four patients over 25 years.
\end{abstract}

Conclusion: In this study, I experienced that a CR was a pivotal influencing factor in those longest survival patients, and traditional medicine was also recommended. Downregulating oncogenic receptors may be useful paradigm and perspective in currently the third line setting of clinical target therapy and in rendering our better understanding of cancer biology.

\section{Introduction}

Chemotherapy is a major skillful of cancer therapy. One of the most important advances in oncology has been increased acceptance of evidence that most patients with disseminated tumors were setted to the protocol of chemotherapy in conjunction with recent targeting oncogenic receptor[1-14],traditional medicine(TCM)and/or adoptive immunotherapy(LAK cells, TIL therapy) $[15,16]$.The experience in Ugandan children with Hodgkin's disease has been excellent [17] and in a study of 14 adults with stage I and II Hodgkin's disease, mostly clinically staged,13 patients (93\%) achieved CR with combination chemotherapy and all were in CR 11 to 94 months after the completion of treatment [18]. Another, a disease-free survivors of 5 years (56.5$59.3 \%$ versus $22-24.3 \%)$ and 10 years (48.9\%) was remarkedly higher rate in those breast cancers with stage III following surgery plus chemotherapy than only surgery. More promising, in a large trials of 48 HER2-positive early breast cancer patients, targeting the adjuvant trastuzumab treatment demonstrated highly favorable outcome. Five year overall survival rates and disease-free survival rates were $95.8 \%$ and $93.8 \%$ respectively [19]. Recently, neratinib was recently approved by FDA for extended adjuvant treatment of ER+/HER2+ breast cancer [20]. Others, advanced or metastatic gastric cancer constitutes the majority of patients in clinical practice. Systemic chemotherapies and combined regimens are currently available, provides palliation and prolongs survival. In particular, high-quality clinical trials on TCM in cancer are generally lacking, except for Kampo medication for Japanese cancer patients [21,22], arsenic trioxide $\left(\mathrm{As}_{2} \mathrm{O}_{3}\right)$ in the role of acute promyelocytic leukemia [23], and cantharidin in treatment of liver cancer [24]. This paper will attempt to place in proper interpretative review from those patients with cancers under remission in this group.

${ }^{\star}$ Correspondence to: George Zhu, Institute of Oncology of George Zhu, 422407, Beijing, China, E-mail: sansan4240732@163.com

Key words: cancer, chemotherapy, traditional medicine, target therapy

Received: July 16, 2018; Accepted: August 10, 2018; Published: August 14, 2018 


\section{Material and Methods}

78 patients with available cancers were concluded in the study during September 1993- May 2018. The sex ratio of male: female was 53:25 respectively. The mean age at onset was 46.9 years ranging from 10 to 79 years. Among age distribution, although there is some uncertain about the type distribution of cancers. It was found 38.1 years as the mean age at onset for lymphoma,44.0 years for liver cancer, while higher mean age at 60.1 years has been shown in lung cancer in this group. The clinical diagnoses in a broad variety of carcinomas consisted of metastatic nasopharyngeal cancer 5 cases, metastatic breast cancer 4 , lung tumors 12 , hepatocellular carcinoma (HCC) 12, stomach cancer 5, hematological malignancies 26 cases (acute leukemias FAB M1 type 2, M2 type 1, acute promyelocytic leukemia 1, chronic myeloid leukemia CML 2,chronic lymphocytic leukemia CLL 1,multiple myeloma 2, lymphoma 16), thyroid cancer 2 , maxillary sinus carcinoma 1 , carcinoma of mandibular sinus 2,laryngeal carcinoma 1,esophagus cancer 1, gallbladder cancer 1, cholangiocarcinoma 1, metastatic oral cancer 1,epidermoid carcinoma 1, metastatic carcinoma 1, replapsed vulvar cancer 1,and other metastatic sternal and spinal (T12) tumor 1 respectively. All other benign neoplasias were not statistically included. The basic chemotherapeutic regimen consisted of vincristine (VCR,12mg/wk), cyclophosphamide (CTX,200-1,000mg/wk) mitomycin $\mathrm{C}(\mathrm{MMC}, 2-4 \mathrm{mg} / \mathrm{wk})$ and 5-fluorouracil (5-Fu, 250-500mg/day). In addition, the additional drug Adriamycin (ADM, 20mg/wk) in lymphoma and metastatic breast cancer, demethylcantharidin in liver cancer and cisplatin (DDP) or interleukin-2(PHA)/gefitinib in lung cancer. The detail prescription of TCM varied among a broad variety of carcinomas (see full text case reports). The criteria of complete remission (CR) and/or partial remission (PR) is according to the rules where physicians have in common with in clinics. Complete remission (CR): there was no more tumor or tumor complete regressed in patients for at least 1 month; Parttial remission: the tumor decreased by more than $50 \%$ in patients for at least 1 month; Stable disease: the tumor decreased by less than $50 \%$ or increased by no more than $25 \%$ in patients; Disease progression: the tumor increased by more than $25 \%$ in patients, or new lesions emerged. The efficacy was evaluated according to the survival time from the day when patients were at onset. The clinical data for liver cancer $[25,26]$ and lung cancer $[27,28]$ were previously described.

\section{Results}

In 78 cancers, the rate of complete remission (CR)was achieved in 33(42.3\%) advanced cancers. All CR patients with advanced cancers was survival over 5 years, 18 cancers was survival 10 years. Another, a short CR was obtained in $12(15.4 \%)$ advanced cancers, the survival time varied from 20 months to 4 years.PR was obtained in 25(34.6\%) patients with a broad variety of carcinoma, while three patients (1 malignant lymphoma, 1 carcinoma of mandibular sinus, 1 metastatic tumor of bone) had survival $12,18+$ and $11+$ years respectively, implicating a longer survivor in patients the survival with tumours. Otherwise, stable disease was 6 cases. Basic characteristics of studied population were summarized in table 1 .

During the schedule of drug administration, all patients were treated with the different dosage of 1 to 4 courses of various combination chemotherapy in conjunction with traditional medicine. In statistically analysis, one patient with nasophyaryngeal cancer, the diplopia and unable version in his eye were recovered to "normal" visual acuity following the combination chemotherapy of VCMF (VCR, CTX, $\mathrm{MMC}$ and 5-Fu) plus traditional medicine. A patient with rodent ulcer
$(8 \times 5 \mathrm{~cm})$ once obtained complete response as to an approach of $5 \% \mathrm{Fu}$ of retinoic acid ointment. A short CR was achieved by the protocol of MFC (MMC;5-Fu; Ara-C/homoharringtonine,CTX)plus cantharidin or cinobufacini drug in 5 advanced gastric cancers. One of them was a long-term survivor for 6 years via mass incision and the combination of MFC with herbs Scutellaria barbata d.don.

In view of cancer types, 10 lymphoma was setted to the major protocol of the combination conventional chemotherapy (COMA, VCR, CTX, MMC or ADM) in conjunction with traditional medicine which to relieve the chemotherapeutic toxicity, and reinforced the efficacy of chemotherapy. One lymphoma was regressed only by prednisone(200\#). Another 4 patients with thumb lymphadenopathy was treated by the use of antibiotics regimen in full dose with antiinflammatory herbal tablets or immunotherapy lymphocyte transfer factor.

In 12 HCC, 6 HCC were treated mainly by 5 -Fu $(500-1,000 \mathrm{mg} /$ day $)$ and TCM. 2 patients obtained CR through cantharidin and traditional medicine. The main protocol of TCM with adjuvant antibiotics regimen and low dose of dexamethasone was given in a primary liver cancer $(\mathrm{AFP}+$, ascites +++ , jaundice +++ , liver tumor $3.2 \times 3.0 \mathrm{~cm})$. One acute promyelocytic leukemia complicated with metastatic liver cancer $(7 \times 4.5 \mathrm{~cm})$ was in CR with all-trans retinoic acid (ATRA) and TCM. The detail prescription of TCM was mentioned before $[25,26]$. In the follow up,one HCC accompanied with colon polyps obtained complete remission via hapatectomy and targeting oncogenic receptor tyrosine kinase inhibitor sorafenib.

Dose intensity has proven to be critical in maximizing chemotherapeutic efficacy for numerous human cancers. Eight other patients with cancers were in remission through small dosage of chemotherapy and TCM or traditional medicine (TCM) alone. There were 4 lung cancers, 1 gallbladder cancer, 1 cholangicarcinoma and 2 thyroid cancers. Among targeting two metastatic lung cancer, one female with lung cancer was given the combination chemotherapy plus targeting oncogenic receptor EGFRvIII gefitinib, which was stable disease for $8+$ months. Thyroid cancer was placed on the primary use of traditional medicine.The crude herbs consisted of sargassum, tangle, Oyster (mussels), Poria cocos, Ophiopogon japonicus, Prunella vulgaris, Taraxacum, Scrophularia ningpoensis, Cremastra appendiculata, Trichosanthes Kirilowii, Sophora subprostrata, Houttuynia cordata, Scutellaria barbata d. don and Oldenlandia diffusa roxb.

The survival times in those patients with remission were less than 1 years 10 cases, 1 to 3 years 22 cases, over 3 to 5 years 12 cases, over 5 to 10 years 11 cases, over 10 to 20 years 13 cases, and over 20 years 7 cases. In differential types of 20 patients with over 10 years survivors, lymphoma occupied 8 cases (40\%). Among 7 patients with over 20 years, lymphoma occupied 3 cases, metastatic breast cancer 2 cases and hepatocellular carcinoma 2 cases.

\section{Case reports}

A 55-year-old woman was diagnosed as having metastatic palatum cancer on November 6,1993 when she presented with tumors both in her cavity of the mouth and neck lymphadenopathy. On examination revealed 2 lymph nodes $(4 \times 3 \mathrm{~cm})$ enlargement in her left neck. A $3 \times 5 \mathrm{~cm}$ mass was found in her palate molle which was covered over uvula palatina. Moreover, the left side of her face also had a thumb lymph nodes palpable.Cures can be achieved by use of combination chemotherapy(VCR,CTX,5-Fu, phytohemagglutinin, PHA) and in 18 years later she died of recurrent episodes of oral cancer. 
Table 1. Patients characteristics. Note: HCC: hepatocellular carcinoma; NPC: metastatic nasopharyngeal cancer; MBC: metastatic breast cancer; AML: acute myeloid leukemia; APL: acute promyelocytic leukemia; CML: chronic myeloid leukemia; CLL: chronic lymphocytic leukemia; MM: multiple myeloma; COMA: CTX,VCR,MMC,ADM; COMF: CTX,VCR,MMC/ ADM,5-FU; VCMF: VCR,CTX,MMC/DDP; 5-FU; COP:CTX, VCR, pred; COFP: CTX,VCR,5-FU,PHA, Pred; DA:DNR,45mg/m², Ara-c 100mg/m²; HA: homoharringtone 1mg X 5days, ara-c 50mg, intramuscle, twice a day; MFC: MMC, 5-FU, Ara-c/H, CTX; COMMB: CTX, VCR, MMC, MTX, Bleomycin; ATRA: all-trans retinoic acid; Pred: prednisone; TCM: traditional medicine; M: male; F: female. ": cases number; "*: treatment in another hospital; ${ }^{* * *}$ include oral cancer 1 , metastatic melanoma 1 , relapsed vulva cancer 1 ,laryngeal cancer 1 , esophagus cancer 1, maxillary sinus carcinoma 1, carcinoma of mandibular sinus 2 , and metastatic bone tumor 2 .

\begin{tabular}{|c|c|c|c|c|c|c|}
\hline Cancer types & Cases No & Sex & $\begin{array}{l}\text { Mean } \\
\text { ages(years) }\end{array}$ & $\begin{array}{l}\text { Treatment } \\
\text { Protocol }\end{array}$ & Response following therapy & $\begin{array}{l}\text { Duration of remission (years) } \\
<11-3>3-5>5-10>10\end{array}$ \\
\hline lymphoma & 16 & M14, F2 & $38.1(13-66)$ & $\begin{array}{l}\text { COMA }(10) * \\
\text { Radiotherapy**(1), } \\
\text { Prednisone (1), } \\
\text { Immunotherapy (4) }\end{array}$ & $\begin{array}{l}\text { CR }(10)^{*} \\
\text { short CR }(2) \\
\operatorname{PR}(4)\end{array}$ & $\begin{array}{lllll}1 & 3 & 2 & 1 & 9\end{array}$ \\
\hline $\mathrm{HCC}$ & 12 & M10, F2 & $44.0(26-63)$ & $\begin{array}{l}\text { a.5-Fu(250-1,000mg/day), VCR, CTX, } \\
\text { MMC, TCM } \\
\text { b.Cantharidin,TCM } \\
\text { c.hepatectomy,sorafenib }\end{array}$ & $\begin{array}{l}\text { CR (8), } \\
\text { Short CR (2), } \\
\text { PR (1), } \\
\text { stable disease (1) }\end{array}$ & $\begin{array}{llll}1 & 4 & 1 & 3\end{array}$ \\
\hline Lung tumors & 12 & M8, F4 & $60.1(40-79)$ & $\begin{array}{l}\text { a.COMF,TCM; } \\
\text { b.DDP,etoposide,gefitinib c.CTX,5- } \\
\text { FU,antitumor capsule; d.TCM alone }\end{array}$ & $\begin{array}{l}\text { CR (2), } \\
\text { short CR (2), } \\
\text { PR (3), } \\
\text { stable disease (5) }\end{array}$ & 43 \\
\hline NPC & 5 & M3, F2 & $51.4(38-75)$ & $\begin{array}{l}\text { a.VCMF,TCM } \\
\text { b.CTX,5-FU, TCM }\end{array}$ & CR (1), shortCR (1), PR (3) & 13 \\
\hline $\mathrm{MBC}$ & 4 & F4 & $31.3(25-41)$ & $\begin{array}{l}\text { a.COMF,,TCM } \\
\text { b.COP,TCM }\end{array}$ & CR (3), PR (1) & 2 \\
\hline Stomach cancer & 5 & M2, F3 & $42.3(35-500$ & $\begin{array}{l}\text { a.MFC,TCM; b.CTX,5- } \\
\text { FU,antitumor capsule }\end{array}$ & CR (1), shortCR (2), PR (2) & 13 \\
\hline AML & 3 & M2, F1 & $4,18,20$ & $\mathrm{DA}^{* *}, \mathrm{HA}, \mathrm{TCM}$ & PR (3) & 3 \\
\hline APL & 1 & M & 31 & $\begin{array}{l}\text { ATRA 80mg/day; } \\
\text { H 1mgx5days; TCM }\end{array}$ & $\mathrm{CR}$ & 1 \\
\hline CML & 2 & M1, F1 & 33,62 & Busulfan,TCM & $\begin{array}{l}\text { CR (1), } \\
\text { short CR (1) }\end{array}$ & $1 \quad 1$ \\
\hline CLL & 1 & M & 58 & Chlorambucil, TCM & $\mathrm{CR}$ & $\begin{array}{c}1 \\
\text { died of stomach cancer }\end{array}$ \\
\hline MM & 2 & M1, F1 & 60,63 & Thalidomide,pred, TCM & $\begin{array}{l}\text { Short CR (1), } \\
\text { PR (1) }\end{array}$ & $1 \quad 1$ \\
\hline $\begin{array}{l}\text { Epidermoid } \\
\text { cancer }\end{array}$ & 1 & F & 72 & $5 \% \mathrm{FU}$ of retinoic acid ointment & PR & 1 \\
\hline Thyroid cancer & 2 & F2 & 54,60 & TCM alone & CR (2) & 1 \\
\hline bile cancer & 1 & $\mathrm{~F}$ & 65 & CTX,5-FU, TCM & $\mathrm{CR}$ & 1 \\
\hline $\begin{array}{l}\text { Cholangio- } \\
\text { carcinoma }\end{array}$ & 1 & M & 72 & MFC (MMC 5-FU) & CR & 1 died of intestinal cancer \\
\hline Others*** & 10 & M8, F2 & $57.5(44-69)$ & $\begin{array}{l}\text { CTX, TCM } \\
\text { COFP, COMMB, TCM }\end{array}$ & CR (1), PR (6) & $\begin{array}{llll}3 & 2 & 1 & 3\end{array}$ \\
\hline
\end{tabular}

A 35-year-old woman was admitted to the hospital because of her relapsed gastric cancer, with recurrent fever for one month duration. In March,1996 she was undergoing surgery in a local hospital due to her tarry stools. At post-operatively, a rodent ulcer $(5 \times 4 \times 2.8 \mathrm{~cm})$ with harden border was detected in lesser curvature of the stomach, accompanied with adjacent metastatic lymph nodes. NHL was diagnosed according to her stomach tissue specimens. The definitive diagnosis of her malignant tumor was based on a provincial tumor hospital. She had a past history of tuberculosis.CR was obtained after small dosage of FMC (5-Fu, MMC, CTX) in combination with cinobufacini intravenously, oral daily demethylcantharidin and traditional herbs. As an outpatient, she had continued to traditional herbs Scutellaria barbata d. don. She was a long-term survivor for 6 years and died of tuberculosis (type IV).

A 58-year-old man was diagnosed as having malignant lymphoma on April 29,1997. He presented his past history of lymph node palpable (a pea size) in right neck region 8 years ago. Later in 1995. A gradually increased thumb lymph node was palpable, accompanied by his left neck metastasis. At physical examination, on admission, showed $4.5 \times 3 \mathrm{~cm}$, $2 \times 3 \mathrm{~cm}, 2 \times 3 \mathrm{~cm}$ palpable lymph node in right neck and a $2 \times 3 \mathrm{~cm}$ lymph node in left neck region. Lymphoma was diagnosed according to his lymph node aspirates. He obtained remission following combination chemotherapy (CTX and 5-Fu) in conjunction with traditional medicine. He was stable disease for 12 years survivor.
A 71-year-old man was admitted to the hospital on December 29,1997 because of the relapse of his lymphoma for 2 months duration. $\mathrm{He}$ once obtained partial remission (PR)using chemotherapy in another tumor hospital.CR was obtained by the main protocol of TCM with small dosage of chemotherapy (CTX,5-Fu). TCM consisted of asparagalus membranaceus, ophiopogon japonicus, asparagus, coix lachryma, paris polyphylla, pseudobulb of appendiculate cremastra, trichosanthes kirilowii, indigowoad leaf, scutellaria barbata d.don, oldenlandia diffusa roxb. He was a survivor of 7 years.

A 62-year-old woman entered the hospital because of her chronic myelocytic leukemia (CML) on October 21,2000. She developed her distended abdomen and splenomegaly 6 months duration. On B ultrasound examination showed that spleen reached to umbilicus, with irregular liver scan. Hemoglobin concentration was $7.0 \mathrm{~g} / \mathrm{L}$. leukocyte count 160,000 (160x109/1) with $19 \%$ blasts and promyelocytes, $28.5 \%$ myelocytes and metamyelocytes. platelet count was $375 \times 109 / 1$. Bone marrow aspirations revealed marked hypercellularity with myeloid hyperplasia. Blast forms (blast and promyelocytes) constituted approximately $19.6 \%$ of all cells, and immature myelocyte and metamyelocytes occupied $28.5 \%$ of all cells. Megakaryocytes was hyperplasia. The diagnosis of CML with accelerate stage was made. CR was obtained after busulfan in conjunctiion with TCM. She was in satisfactory health until 4 years before admission. When she was 
found to be splenomegaly. At that time, WBC 120x109/l. The high percentage of blast cells corresponded to the beginning of her relapse. Oral busulfan was administered in the following a total dose of $120 \mathrm{mg}$, with CR again. She was survivor for near 8 years.

A 65-year-old woman was diagnosed as having gallbladder cancer in July 20,2002. She developed symptoms of lancinating abdominal pain and intensive distended abdomen and tender with muscle defense in upper quadrants. Ascites+++. On CT scan demonstrated that her gallbladder was dilation, with irregular thick cholecystic inner wall, and many nodules were found in the cavity of bile. She was given the combination of TCM with small dosage of 5-Fu and CTX drugs. Three months later, in view of improvement of her general symptoms, she obtained CR. She is in health during follow up of 15 years.

A 25-year-old man was admitted to the hospital on October 4,2003 because of his relapsed malignant lymphoma. He complained of his enlarged lymph nodes once regression using herbs in 1997.He had a past experience of combination chemotherapy due to lymph node enlargement in bilateral neck, which was diagnosed as having malignant lymphoma (B cell type). In September, 2003, he developed his nasopharynx the markedly inflamed redness, swelling, and with obstruction. At physical examination revealed 5 firm lymph nodes to varying degree in size of a pea to a walnut in his left neck, and 2 palpable lymph nodes $(1.5 \times 2 \mathrm{~cm})$ in his right neck. A $3 \times 4.5 \mathrm{~cm}$ mass was found in his soft palate molle which was flecked with small red ulcer patches.CR can be achieved by use of antibiotics regimen in full dose and combination chemotherapy (VCR, CTX, 5-Fu, MMC), the tumors was disappeared as like the mouth of health individuals. He was a 3 years of survivors.

A 72-year-old man was a admitted to the hospital due to his jaundice cholangiocarcinoma on September 2, 2004.He had a history of bad cough two months ago, followed by a progressive general jaundice, conspicuous weight loss, no appetite and urine icterus. On CT examination showed his complete obstructive choledodus and his cholangiectasis due to the cause of obstructive tumor $(1.5 \times 2 \mathrm{~cm})$. He was given the treatment of antibiotics in full dose, combination chemotherapy(5-Fu,CTX,MMC) with TCM. CR was achieved two months later, and as an outpatient, he was to be continued the traditional herbs.Traditional medicine consisted of astragalus membranaceus,ophiopogon japonicas,asparagus,poria cocos,lyceum chinenses, wheat sprout,salvia bowleyana,scutellaria baicalensis,Artemisia capillaries,gardenia jasminoides,hypericum japonicum,houttuynia cordata,scutellaria barbata d.don,oldenlandia diffusa roxb.

A 38-year-old woman was admitted to the hospital on September 26, 2004 due to palpable lymph nodes in her neck for 1+ year duration. One year before admission to the hospital she accidentally noted lymphadenopathy in her bilateral neck region, and lymph node enlargement regressed with unknown drugs. In July,2003, she presented no efficacy following treatment because of her lymphadenopathy relapsed. When examined, there were 6 lymph nodes palpable in her left neck, with varied degree in size of a pigeon's egg to a pea or thumb size. Scrofula with caseous necrosis was diagnosed according to her lymph node aspirates. TB-Ab negative. Remission was obtained through antiTB regimen in combination with TCM.TCM consisted of traxacum, honeysuckle, ophiopogon japonicus, asparagus, mussels, coix lachryma, houttuynia cordate, scutellaria barbata $d$. don, oldenlandia diffusa roxb. In the follow up, she was a 15 years survivor.

A 58-year-old man was diagnosed as having chronic lymphocytic leukemia (CLL)on January 16,2011 because of recent leukocyte counts elevated to $118 \times 109 / 1$.The patient complained of his leukocytosis(67$97 \times 109 / 1)$ for more than one month duration. He was treated with hydroxycarbamide in another hospital and leukocytosis declined to $27 x 109 / 1$. The most common physical signs revealed two thumb lymph nodes palpable in his left neck. Hemogram: Hemoglobin concentration (Hb)was 87g/l.leukocyte count (WBC)123.88x109/1. The leukocyte differential count:9\% segmented neutrophils, $90 \%$ small lymphocytes. The platelet count 131x109/1. Bone marrow aspiration revealed hypercellularity. Bone marrow differential count:14\% myeloid,7.2\% erythroid, approximately $76.8 \%$ of predominant cell was small lymphocytes. The diagnosis of CLL was made.CR was obtained by the use of chlorambucil tablets and traditional medicine. On April 9,2011, repeat hemogram: Hb 112g/l, WBC 13.6x109/l, plt 128x109/l. On May 5 and July 28,2011, Hb 104-112g/l; WBC 9.54-10.1x109/1, with a leukocyte differential count of $26.2 \%$ mature neutrophils and $63.8 \%$ lymphocytes; plt 101-112x109/1 respectively. Bone marrow aspirates on May14,2011 revealed normal cellularity.B one marrow differential count:34\% myeloid,31.2\% erythoid,33.6\% lymphocytes. As an outpatient, he continued traditional herbs. He was well until on October 15,2011 while an attack of stomach pain and tarry stools was admitted to another hospital. Routine hematologic studies at that time, $\mathrm{Hb} 69 \mathrm{~g} / \mathrm{l}$; WBC $7.3 \times 109 / 1$ with $70 \%$ mature neutrophils and $28 \%$ lymphocytes; plt 188x109/1. Repeat bone marrow aspirates on October 15,2011 revealed normal cellularity. Bone marrow differential counts:50\% myeloid, $21 \%$ erythroid, $27.5 \%$ lymphocytes. He died of another stomach cancer.

\section{Discussion}

In this study, a series of the long follow up of patients with cancers were reported. I experienced that a CR was a pivotal influencing factor in those longest survival patients, and traditional medicine was also recommended.

The traditional combination chemotherapy program for lymphomas of favorable histologic type has been CVP (CTX,VCR,Pred) given at 21-days intervals[29]. Cyclophosphamide, vincristine, procarbazine, and prednisone (COPP) were used in the NCI study for patients with nodular mixed and nodular histocytic lymphomas [30]. More intensive CVP programs with the addition of Adriamycin or bleomycin, or both, known as BACOP or CHOP-bleo, resulted in overall complete remission rates for patients with diffuse lymphomas ranging from $48 \%$ to $89 \%$ [31-34].The NCI program of this 5-drug program, complete remission rates with this approaches has ranged from $48 \%$ to $94 \%$ [33]. In this study, the $\mathrm{CR}$ rates was $63 \%$ in 16 lymphomas, $6 \mathrm{CR}$ were used by CVP or COMA regimens.

The use of chemotherapy to treat stomach cancer has no firmly established standard of care [35]. Some drugs used in stomach cancer treatment have included:5-Fu(fluorouracil), doxorubicin(Adriamyci $\mathrm{n}$ ), mitomycin $\mathrm{C}$ and most recently oxaliplatin, irinotecan in various combination. The relative benefits of these different drugs, alone or in combination, are unclear [36].There are evidence supporting that clinical researches are exploring the benefits of giving chemotherapy as adjuvant therapy for surgery to destroy remaining cancer cells [37]. In recent analyses of definitive surgery followed by adjuvant radio chemotherapy (5-Fu/leucovorin LV regimens) for patients with gastric cancer, Liu and Ahmed [38] reported that 59.3\%(48/81) patients survived $>3$ years, $18.5 \%(15 / 81)$ patients survived 5 or more years. Eighteen out of $81(22.2 \%)$ patients are still alive with a medium survival of 142 months(57-196 months). In this study, 5 patients with gastric cancer obtained a short CR through MFC regimen plus cinobufacini and cantharidin drugs. One relapsed gastric cancer survived over 
6 years after surgery and adjuvant chemotherapy. More recent, treatment with HER2 inhibitor, trastuzumab, has been demonstrated to improve overall survival in inoperable locally advanced or metastatic gastric carcinoma overexpressing the HER2 [37]. Oncogenic receptor HER2 [39] is overexpressed in 13-22\% of patients with gastric cancer [40,41].Tanz and colleagues [42] reported two HER2- positive metastatic gastric adenocarcinoma who favorably responded to second line chemotherapy(FOLFIRI, irinotecan plus 5-Fu) with trastuzumab continuation following progressive disease to first line treatment containing trastuzumab, implicating trastuzumab continuation in metastatic HER positive gastric cancer is safe, practical and improve survival.

Oncogenic EGFR mutations are found in $10 \%$ to $35 \%$ of lung adenocarcinomas, with predominants in a subset of patients with non-small cell lung cancer (NSCLC) [43-49]. These mutations, which commonly occur as either small in-frame deletions in exon 19 or point mutations T790M or L858R in exon 21 within the EGFR tyrosine kinase domain, confer constitutive activity and sensitivity to EGFR tyrosine kinase inhibitor (TKI) [49,50]. Konduri and colleagues [51] reported five patients with metastatic lung cancer whose tumors harbored EGFR fusion,most commonly RAD5,are recurrent in lung cancer. Four of whom were treated with EGFR TKI erlotinib with documented antitumor response for 5,6,8, and 20 months respectively. An early EGFR TKI trial randomized patients with EGFR mutation positive stage IIIb or IV adenocarcinoma to treatment with afatinib or gemcitabine and cisplatin, treatment with afatinib prolonged progression free survival to 11.0 months as opposed to 5.6 months with gemcitabine and cisplatin [7]. In a total of 65 lung cancers with EGFRmut (exon19 del/L858R, no T790M), after INC 280 plus gefitinib, partial remission (PRs) were obtained in 12/65 evaluable patients (ORR $18 \%)$ and 40/65 (62\%) patients had stable disease [52]. Central nervous system(CNS) metastases are common in patients with non-smallcell lung cancer(NSCLC). Osimertinib has shown systemic efficacy in patients with CNS metastases, and early clinical evidence shows efficacy in the CNS. In the phase II trials of 50 patients with T790Mpositive advanced NSCLC, confirmed CNS ORR (objective response rate) and DCR (disease control rate) were 54\% (27/50) and $92 \%$ (46/50) respectively. Median follow-up for CNS PFS (progression-free survival) was 11 months. Osimertinib (80mg) demonstrated clinically meaningful efficacy against CNS metastases [53]. In the phase III trial of 419 patients with advanced T790M positive NSCLC with osimertinib vs platinum based therapy, progression free survival in the osimertinib group was 8.5 months, compared to the platinum-based therapy group at 4.2 months [6]. Otherwise, targeting oncogenic ALK inhibitors Crizotinib (250mg, twice a day) [54], and Alectinib(600mg,orally twice daily,second-generation ALK inhibitor, better efficacy and better tolerability) also provented lung cancer progression and delayed the time to brain metastases according to the results of the phase III ALEX trial presented at the 2017 ASCO Annual Meeting [55]. Serra [56] reported the clinical response of a lapatinib-based therapy in lung metastatic lesions of a Li-Fraumeni syndrome patient with oncogenic HER2V659E mutation and an EGFR-exon 20 insertion. A symptomatic and radiologic clinical response was achieved using oral daily lapatinib at a dose of $1,000 \mathrm{mg}$ in combination with intravenous weekly paclitaxel $80 \mathrm{mg} / \mathrm{m} 2$, lately, trastuzumab initial dose of $8 \mathrm{mg} / \mathrm{kg}$ intravenously, and then followed by $6 \mathrm{mg} / \mathrm{kg}$ every three weeks.In total, the clinical benefits lasted over 9 months. In Cuba, CimaVax-EGF, promising, an active vaccine targeting EGF as the major ligand of oncogenic EGFR, it is in use as a cancer therapy against non-small cell lung cancer (NSCLC) $[57,58]$. In this study, we use gefitinib in keeping stable disease for $8+$ months in a woman with lung adenocarcinoma and using gefitinib in more patients are under investigation.

Interesting, in an APL complicated with secondary HCC. It has been demonstrated previously that nuclear RARB has been shown to be rearranged as a result of insertion of HBV sequences [59]. Recent promising, these oncogenic receptor derivatives [23,60-62], in addition to oncogenic pml/RARA, oncogenic TBL1XR1-RARB [63], and NUP98/RARG [64,65], and oncogenic PML-RARG [66] were also detected in APL rare cases. The involvement of RARB may explain why the disappearance of malignant hepatic tumour cells via the use of ATRA agent. In this case, ATRA $(80-100 \mathrm{mg} /$ day) was resistance to the relapse episode. In the presence of genetic mutation in RARA LBD and the PML-B2 domain of PML-RARA, one explanation for ATRA resistance is that the N-CoR/ SMAT- corepressor complex tightly interact with pml/RARA or PLZF, even under pharmacological concentration of ATRA, so that transcriptional de-repression cannot occur at RARA target gene promoter, ATRA binding LBD impaired, degradation of $\mathrm{pml} / \mathrm{RARA}$ by proteasome pathway are inhibited [23, 67]. In addition, new emerging aberrant pml/RARA in relapsed APL returned to act as a constitutive transcriptional repressor[1,23,58,67-83] by pertubing normal retinoid signaling and RAR function, suppressing (the blockade of ) differentiation [23], possessing an altered specify for DNA response element, these DNA recognition changes target a distinct set of "neoplastic" genes that differ from the genes normally targeted by normal RARA. Previous studies uncovered that the ATRA and 13-cis forms of retinoic acid, two isomers of RA, are equally effective inhibiting proliferation [84]. In literature alternative strategy, an APL obtained CR after treatment with 13-cis retinoic acid first and repeated CR with ATRA in relapse [85]. And more, 80\% (4/5) CR in newly APL and 33\% (4/12) CR in relapsed APL were achieved after treatment with 9-cis retinoic acid (LGD1057) alone [86]. Nowadays, a lot of cohort trials, 61.5\% (24/39) achieved CR using tamibarotene including 5 newly APL and 13 relapse APL twice or more [87-93]. Among 269 APL with CR underwent maintenance random, four year relapse-free survival rate was $84 \%$ (ATRA) and $91 \%$ (Tamibarotene). In 52 high risk patients, this became significant (50\% for ATRA, $87 \%$ for tamibarotene) [92]. In comparative analysis among those relapsed APL [94], 80\%(28/35) achieved CR and 22.86\% CRm in tamibarotene- ATO versus $54.2 \%$ (19/35)CR with only $2.86-3.7 \%$ CRm in ATRA and ATO regimen [94]. In particular, an appreciable benefits of tamibarotene-ATO regimen might occur at significantly lower frequency of leukocytosis with development of retinoic acid syndrome, an important adverse reaction during treatment of APL. Thus, Tamibarotene demonstrated more efficacy in both untreated APL patients and relapsed who have been treated ATRA and chemotherapy, especially as novel strategy in relapsed APL in Japan and others [9496]. This is encouraging perspective.

\section{Conflicts of interest}

The author declares that there is no conflict of interest regarding the publication of this paper.

\section{References}

1. Zhu G, Saboor-Yaraghi AA, Yarden Y, Santos J, Neil JC (2016) Downregulating oncogenic receptor: From bench to clinic. Hematol Med Oncol 1: 30-40.

2. Zhu G, Saboor-Yaraghi AA, Yarden Y (2017) Targeting oncogenic receptor:from molecular physiology to currently the standard of target therapy. Advance Pharmaceutical Journal 2: 10-28.

3. van den Heuvel CNAM, Das AI, de Bitter T, Simmer F, Wurdinger T, et al. (2018) Quantification and localization of oncogenic receptor tyrosine kinase variant transcripts using molecular inversion probes. Scientific Reports 8: 7072. 
4. Toledo RA, Garralda E, Mitsi M, Pons T, Monsech J, et al. (2018) Exome sequencing of plasma DNA portrays the mutation landscape of colorectal cancer and discovers mutated VEGFR2 receptors as modulators of anti-angiogenic therapies. Clin Cancer Res 24: 3550-3559.

5. Cross DA, Ashton SE, Ghiorghiu S, Eberlein C, Nebhan CA, et al. (2014) AZD9291, an irreversible EGFR TKI, overcomes T790M-mediated resistance to EGFR inhibitors in lung cancer. Cancer Discov 4: 1046-1061. [Crossref]

6. Conterato AJ, Belanger AR, Yarmus LB, Akulian JA (2017) Update on NSCLC tissue acquisition,processing, and profiling in the molecular age. Hematology Med Oncology 2: $1-8$.

7. Pai SK, Rosenberg JE, Hoffman-Censits JH, Berger R, Quinn DI (2018) Efficacy of BGJ398, a fibroblast growth factor receptor 1-3 inhibitor,in patients with previously treated advanced urothelial carcinoma with FGFR3 alteration. Cancer Discov 8: 812821.

8. Liu J, Sareddy GR, Zhou M, Viswanadhapalli S, Li X, et al. (2018) Differential effects of estrogen receptor beta isoforms on glioblastoma progression. Cancer Res 78: 31763189.

9. Weir HM, Bradbury RH, Lawson M, Rabow AA, Butter D, et al. (2016) AZD9496.An oral estrogen receptor inhibitor that block the growth of ER-positive and ESR1-mutant breast tumors in preclinical models. Cancer Res 76: 3307-3318.

10. Went DC, Kocherginsky M, Tonsing-Carter EY, Dolcen N, Hosfield DJ (2018) Discovery of a glucocorticoid receptor (GR) activity signature using selective GR antagonism in ER-negative breast cancer. Clin Cancer Res 24: 3433-3446.

11. Reddy JA, Allagadda VM, Leamon CP (2005) Targeting therapeutic and imaging agents to folate receptor positive tumors. Curr Pharm Biotechnol 6: 131-150.

12. Kalli KR, Block MS, Kasi PM, Erskine CL, Hobday TJ, et al. (2018) Folate receptor alpha peptide vaccine generates immunity in breast and ovarian cancer patients. Clin Cancer Res.

13. Zhu G, Saboor-Yaraghi A, Dharmadhikari D, Baer J (2017) A pilot study of chemotherapy and traditional plant medicine in hematology malignancy:report of thirty-four cases. Hematology Med Oncology 2: 2.

14. Zhu G (2018) EpCAM-an old cancer antigen,turned oncogenic receptor and its targeting immunotherapy. Universal Journal of Pharmaceutical Research(ujpr) 3: 43-48.

15. Rosenberg SA (1985) Observation on the sysyemic administration of autologous lymphokine-activated killer cells and recombinant interleukin-2 in patients with metastatic cancer. $N$ Engl J Med 313:1485

16. Forget MA, Haymakere C, Hess KR, Meng YJ, Creasy C, et al. (2018) Prospective analysis of adoptive TIL therapy in patients with metastatic melanoma:response,impact of anti-CTLA4, and biomarkers to predict clinical outcome. Clin Cancer Res.

17. Olweny CL, Katongole-Mbidde E, Kiire C, Lwanga SK, Magrath I, et al. (1978) Childhood Hodgkin's disease in Uganda: a ten year experience. Cancer 42: 787-792. [Crossref]

18. Lauria F, Baccarani M, Fiaccini M, Mazza P, Tura S (1979) Combination chemotherapy in stages I or II Hodgkin's disease. Lancet 2: 1072-1073. [Crossref]

19. Kato M, Sakuyama A, Matsutani T, Minato H (2015) Efficacy of Trastuzumab therapy in HER2-positive early breast cancer patients in our clinic. Proceedings of BIT's 8th Annual World Cancer Congress pp. 301.

20. Singh H, Walker AJ, Amiri-Kordestani L, Cheng J, Tang S, et al. (2018) US Food and Drug Administration Approval:Neratinib for extended adjuvant treatment of early stage HER2-positive breast cancer. Clin Cancer Res 15: 3486-3491.

21. Takeda T, Yamaguchi T, Yaegashi N (2012) perceptions and attitudes of Japanese gynecologic cancer patients to Kampo (Japanese herbal) medicines. Int J Clin Oncol 17: 143-149.

22. Ito A, Munakata K, Imazu Y, Watanabe K (2012) First nationwide attitude survey of Japanase physicians on the use of traditional Japanese medicine (Kampo) in cancer treatment. Evidence-Based Complementary and Alternative Medicine, Hindwai.

23. Zhu G (2013) Novel treatment of acute promyelocytic leukemia: Asâ,,Oâ, $f$, retinoic acid and retinoid pharmacology. Curr Pharm Biotechnol 14: 849-858. [Crossref]

24. Zhang Q, Zhu G (2017) The pathological pattern of seven malignant cancers following Demethylcantharidin. Advance Pharmaceutical Journal 2: 243-247.

25. Zhu G, Musumecci F, Byrne P, Gupta D, Gupta E (2017) Treatment of advanced hepayocelluar carcinoma(HCC)with the combined protocol of chemotherapy 5 -fluorouracil and traditional medicine:report of ten cases. Clin Trials Pathol Case Stud 2: 61-65.
26. Zhu G, Musumecci F, Byrne P, Gupta D, Gupta E (2017) Role of traditional herbal medicine in the treatment of advanced hepatocellular carcinoma (HCC): past and future ongoing. Advance Pharmaceutical Journal 2: 115-120.

27. Zhu G, Musumecci F, Byrne P, Gupta D, Gupta E, Baer J (2017) A pilot study of lung cancer following chemotherapy and traditional medicine:report of 12 cases. Lungs and Breathing 1: 1-4.

28. Zhu G, Musumecci F, Byrne P, Gupta D, Gupta E, Baer J (2017) Clinical trials of lung cancer after chemotherapy and traditional medicine (12 cases). Advance Pharmaceutical Journal 2: 199-203.

29. Bonadonna G, Lattuada A, Monfardini S (1979) Combined radiotherapy-chemotherapy in localized non-Hodgkin's lymphomas: five-year results of a randomized study. In Adjuvant Therapy of Cancer II. Jones SE, Salmon SE, Grune, Stratton (Eds) New York pp: $145-153$.

30. Anderson T, Bender RA, Fisher RI, DeVita VT, Chabner BA, et al. (1977) Combination chemotherapy in non-Hodgkin's lymphoma: results of long-term followup. Cancer Treat Rep 61: 1057-1066. [Crossref]

31. Skarin AT, Rosenthal DS, Moloney WC (1977) Combination chemotherapy of advanced non-Hodgkin's lymphoma with bleomycin, Adriamycin, cyclophosphamide, vincristine, and prednisone(BACOP). Blood 49: 759-769.

32. Rodriguez V, Cabanillas F, Burgess MA, McKelvey EM, Valdivieso M, et al. (1977) Combination chemotherapy ("CHOP-Bleo") in advanced (non-Hodgkin) malignant lymphoma. Blood 49: 325-333. [Crossref]

33. Schein PS, DeVita VT Jr, Hubbard S (1976) Bleomycin, Adriamycin, Cyclophosphamide, Vincristine, and Prednisone (BACOP) combination chemotherapy in the treatment of advanced diffuse histocytic lymphoma. Ann Intern Med 85: 417-422.

34. Case DC (1979) Combination chemotherapy of advanced diffuse non-Hodgkin's lymphoma:results of cyclophosphamide,Adriamycin,vincristine,prednisone, and bleomycin (CHOP-bleo). J Maine Med Assoc 70: 348-352.

35. Wagner AD, Syn NL, Moehler M, Grothe W, Yong WP, et al. (2017) Chemotherapy for advanced gastric cancer. Cochrane Database Syst Rev 8: CD004064. [Crossref]

36. Scartozzi M, Galizia E, Verdecchia L, Berardi R, Antognoli S, et al. (2007) Chemotherapy for advanced gastric cancer: across the years for a standard of care. Expert Opin Pharmacother 8: 797-808. [Crossref]

37. Orditura M, Galizia G, Sforza V, Gambardella V, Fabozzi A1, et al. (2014) Treatment of gastric cancer. World J Gastroenterol 20: 1635-1649. [Crossref]

38. Liu JL, Ahme S (2018) Long term survival of patients with gastric cancer treated with adjuvant radio-chemotherapy: proposal of a prognostic index with implication for treatment modification. Oncol Res Rev 1: 1-5.

39. Skrypek N (2015) The oncogenic receptor ErbB2 modulates gemcitabine and irinotecin SN-38 chemoresistance of human pancreatic cancer cells via hCNT1 transporter and multidrug resistance associated protein MRP-2. Oncotarget 6: 10853-10861.

40. Meza-Junco J, Au HJ, Sawyer MB (2011) Critical appraisal of trastuzumab in treatment of advanced stomach cancer. Cancer Manag Res 3: 57-64. [Crossref]

41. Fusco N, Rocco EG, Del Conte C, Pellegrini C, Bulfamante G, et al. (2013) HER2 in gastric cancer: a digital image analysis in pre-neoplastic, primary and metastatic lesions. Mod Pathol 26: 816-824. [Crossref]

42. Tanz R, Mahfoud T, Alami EI, Bazine A, Errihani H, et al. (2018) Is there any advantage from continuation of trastuzumab beyond progression in metastatic her positive gastric cancer?. Hematol Med Oncol 3: 2.

43. Gabitova L, Gorin A, Astsaturov I (2014) Molecular pathways: sterols and receptor signaling in cancer. Clin Cancer Res 20: 28-34. [Crossref]

44. Shimizu N, Kondo I (1982) Hyperproduction of EGF receptor in human A431 cel is regulated by a translocation chromosome,t(7;11)(p22;q23). Cytogenetics and Cell Genetics 32: 316-317.

45. Merlino GT, Xu YH, Ishii S, Clark AJ, Semba K, et al. (1984) Amplification an enhanced expression of the epidermal growth factor receptor gene in A431 human carcinoma cells. Science 224: 417-419.

46. Ullrich A, Coussens J, Hayflick JS, Dull TJ, Gray A, et al. (1984) Human epidermal growth factor receptor cDNA sequence and aberrant expression of the amplified gene in A431 epidermoid carcinoma cells. Nature 309:418-425.

47. Miltra S, Han S, Soderstram K, Wong A (2018) Preferential expression of an oncogenic receptor in brain tumor stem cells:identification and targeting using an engineered antibody. In: Proc Am Assoc Cancer Res. Cancer Res p. 72. 
48. Hembrough T, Thyparambil S, Liao WL, Darfler M, Krizman D, et al. (2012) Quantitative multiplexed SRM analysis of oncogenic receptors in FFPE colorecta carcinoma tissue. AACR 103rd Annual Meeting Chicago, IL. Cancer Res 72: 5537.

49. Lee JC, Vivanco I, Beroukhim R, Huang JH, Fenh WL, et al. (2006) Epidermal growth factor receptor activation in glioblastoma through novel missense mutations in extracellular domain. PLoS medicine 3: e485.

50. Godin-Heymann N, Bryant I, Rivera MN, Ulkus L, Bell DW, et al. (2007) Oncogenic activity of epidermal growth factor receptor kinase mutant alleles is enhanced by the T790M drug resistance mutation. Cancer Res 67: 7319-7326.

51. Konduri K, Gallant JN, Chae YK, Giles FJ, Gitlitz BJ4, et al. (2016) EGFR Fusions as Novel Therapeutic Targets in Lung Cancer. Cancer Discov 6: 601-611. [Crossref]

52. Wu YL, Kim DW, Felip E, Zhang L, Liu X, et al. (2016) Phase II safety and efficacy results of a single-arm ph ib/II study of capmatinib(INC 280) + gefitinib in patients(pts) with EGFR-mutated(mut),cMET-positive(cMET+) non-small cell lung cancer(NSCLC). J Clin Oncol 34: 9020-9020.

53. Goss G, Tsai CM, Shepherd FA, Ahn MJ, Bazhenova L, et al. (2018) CNS response to osimertinib in patients with T790M-positive advanced NSCLC: pooled data from two phase II trials. Ann Oncol 29: 687-693. [Crossref]

54. Shun $\mathrm{Lu}$ (2016) Phase 3 study of first-line crizotinib vs pemetrexed/cisplatin or carboplatin (PCC) in East Asian patients(pts) with ALK+ advanced non-squamous nonsmall cell lung cancer(NSCLC). J Clin Oncol 34: 9058-9058.

55. Shaw AT, Peters S, Mok T, (2017) Alectinib versus Crizotinib in treatment-naive advanced ALK positive non-small cell lung cancer(NSCLC):primary results of the Global Phase III ALEX Study. J Clin Oncol 35: 08.

56. Serra V, Vivancos A, Puente XS, Felip E, Silberschmidt D, et al. (2013) Clinical response to a lapatinib-based therapy in a Li-Fraumeni syndrome patient with a novel HER2V659E mutation. Cancer Discov 3: 1238-1244.

57. Rodríguez PC, Rodríguez G, González G, Lage A (2010) Clinical development and perspectives of CIMAvax EGF, Cuban vaccine for non-small-cell lung cancer therapy. MEDICC Rev 12: 17-23. [Crossref]

58. Gonzalez G, Crombet T, Lage A (2011) Chronic vaccination with a therapeutic EGFbased cancer vaccine: a review of patients receiving long lasting treatment. Curr Cancer Drug Targets 11: 103-110.

59. De H, Marchio A, Tiollais P, Dejean A (1987) A novel steroid thyroid hormone receptor- related gene inappropriately expressed in human hepatocellular carcinoma Nature 330: 667

60. Marinelli A, Bossi D, Pellicci PG, Minucci S (2007) A redundant oncogenic potential of the retinoic acid receptor (RAR) alpha,beta and gamma isoforms in acute promyelocytic leukemia. Leukemia 21: 647-650.

61. Rietveld LE, Caldenhoven E, Stunnenberg HG (2001) Avian erythroleukemia: a model for corepressor function in cancer. Oncogene 20: 3100-3109. [Crossref]

62. Hauksdotti H, Privalsky ML (2001) DNA recognition by the aberrant retinoic acid receptors implicated in human acute promyelocytic leukemia. Cell Growth Differ 12: 85-98.

63. Osumi T, Tsujimoto SI, Tamura M, Uchiyama M, Nakabayashi K, et al. (2018) Recurrent RARB-translocations in acute promyelocytic leukemia lacking RARA translocation. Cancer Res 78: 4452-4458.

64. Such E, Cervera J, Valencia A, Barragán E, Ibañez M, et al. (2011) A novel NUP98/ RARG gene fusion in acute myeloid leukemia resembling acute promyelocytic leukemia. Blood 117: 242-245. [Crossref]

65. Such E, Cordón L, Sempere A, Villamón E, Ibañez M, et al. (2014) In vitro all-trans retinoic acid sensitivity of acute myeloid leukemia blasts with NUP98/RARG fusion gene. Ann Hematol 93: 1931-1933. [Crossref]

66. Ha JS, Do YR, Ki CS, Lee C (2017) Identification of a novel PML-RARG fusion in acute promyelocytic leukemia. Leukemia 31: 1992-1995. [Crossref]

67. Tomita A, Kiyoi H, Nao T (2013) Mechanisms of action and resistance to all-trans retinoic acid (ATRA) and arsenic trioxide(As2O3) in acute promyelocytic leukemia. Int J Hemat 97: 717-725.

68. Grignani F, Ferrucci P, Testa U (1993) The acute promyelocytic leukemia-specific PML-RARa fusion protein inhibits differentiation and promotes survival of myeloid precursor cells. Cell 74: 423-431.

69. Rousselot P, Hardas H, Patel A (1994) The PML-RARa gene product of the $t(15 ; 17)$ translocation inhibits retinoic acid-induced granulocytic differentiation and mediated transactivation in human myeloid cells. Oncogene 9: 545-551.
70. He LZ, Guidez F, Tribioli C, Peruzzi D, Pandolfi PP (1998) Distinct interactions of PML-RARa and PLZF-RARalpha with co-repressors determine differential response to RA in APL. Nature Genetics 18: 126-135.

71. Kitareewan S, Pitha-Rowe I, Sekula D (2002) UBEIL is a retinoid target that triggers $\mathrm{PML} / \mathrm{RARa}$ alpha degradation and apoptosis in acute promyelocytic leukemia. Proc Nat Acad Sci USA 99: 3806-3811.

72. Segalla S, Rinaldi L, Kalstrup-Nielsen C (2003) Retinoic acid receptor alpha fusion to PML affects its transcriptional and chromatin-remodeling properties. Mol Cell Biol 23: $8795-8808$.

73. Jing Y (2004) The PML-RARalpha fusion protein and targeted therapy for acute promyelocytic leukemia. Leuk Lymphoma 45: 639-648. [Crossref]

74. Carbone R, Botrugno OA, Ronzoni S, Insinga A, Di Croce L, et al. (2006) Recruitment of the histone methyltransferase SUV39H1 and its role in the oncogenic properties of the leukemia-associated PML-retinoic acid receptor fusion protein. Mol Cell Biol 26 : 1288-1296. [Crossref]

75. Nasr R, Guillemin MC, Ferhi O (2008) The eradication of acute promyelocytic leukemiainitiating cells through PML-RARA degradation. Nature Medicine 14: 1333-42.

76. Marstrand TT, Borup R, Willer A, Borregaard N, Sandelin A, et al. (2010) A conceptual framework for the identification of candidate drugs and drug targets in acute promyelocytic leukemia. Leukemia 24: 1265-1275. [Crossref]

77. Lallemand-Breitenbach V, de Thé H (2010) A new oncoprotein catabolism pathway. Blood 116: 2200-2201. [Crossref]

78. Rosen M, Privalsky ML (2011) Thyroid hormone receptor mutations in cancer and resistance to thyroid hormone:perspective and prognosis. Journal of thyroid research, Hindawii.

79. Podhorecka M, Macheta A (2013) Acute promyelocyticleukemia-modern approach to disease pathogenesis and differentiation treatment. Postepy Hig Med Dosw 67: 1083-1089.

80. Dos Santos GA, Kats L, Pandolfi PP (2013) Synergy against PML-RARa targeting tran scription,proteolysis, differentiation, and self-renewal in acute promyelocytic leukemia. J Exp Med 210: 2793-2802.

81. Humbert M (2014) The tumor suppressor gene DAPK2 is induced by myeloid transcription factor pu.1 and c.EBPa during granulocytic differentiation but repressed by PML-RARa in APL. J Leuk Biol 95: 83-93.

82. De Braekeleer E, Douet-Guilbert N, De Braekeleer M (2014) RARA fusion genes in acute promyelocytic leukemia: a review. Expert Rev Hematol 7: 347-357. [Crossref]

83. Noguera NS, Piredda ML, Taulli RC, Lo-Coco F (2016) PML/RARa inhibits PTEN expression in hematopoietic cells by competing with PU.1 transcriptional activity. Oncotarget 7: 66386-66397.

84. Chomienne C, Ballerini P, Balitrand N, Daniel MT, Fenaux P, et al. (1990) All-tran retinoic acid in acute promyelocytic leukemia II.In vitro studies, structure-function relationship. Blood 76: 1710 .

85. Haferiach T, Lffler H, Glass B, Gassmann W (1993) Repeated complete remission in a patient with acute promyelocytic leukemia after treatment with 13-cis-retinoic acid first and with all-trans-retinoic acid in relapse. Clin Investig 71: 774-779.

86. Soignet SL, Benedetti F, Fleischauer A, Parker BA, Truglia JA, et al. (1998) Clinica study of 9-cis retinoic acid (LGD) in acute promyelocytic leukemia. Leukemia 12 1518-1521.

87. Tobita T, Takeshita A, Kitamura K (1997) Treatment with a new synthetic retinoid, Am80, of acute promyelocytic leukemia relapsed from complete remission induced by all-trans retinoic acid. Blood 90: 967-73.

88. Takeuchi M, Yano T, Omoto E, Takahashi K, Kibata M (1998) Relapsed acute promyelocytic leukemia previously treated with ATRA: clinical experience with a new synthetic retinoid, Am80. Leuk lymphoma 31: 441-451.

89. Takeuchi M, Yoshida I, Takahashi K (2003) Long-term follow up of re-induction with a new synthetic retinoid, Am-80, for relapse of acute promyelocytic leukemia previously treated with all-trans retinoic acid: results of 7 cases from a single institute. Rinsho Ketsueki 44: 1069-1073.

90. Takeshita A, Shinagawa K, Adachi M, Ono T, Kiguchi T, et al. Tamibarotene for the treatment of acute promyelocytic leukemia. Expert opinion on orphan drugs 2: 961-969.

91. Naoe T (2014) Tamibarotene for the treatment of acute promyelocytic leukemia Hematology 71: 96-69.

92. Shinagawa K, Yanada M, Sakura T (2014) Tamibarotene as maintenance therapy for acute promyelocytic leukemia: results from a randomized controlled trial. J Clin Oncol 32: 3729-3735. 
93. Sanford D, Lo-coco F, Sanz MA, Bona ED, Coutre S, et al. (2015) Tamibarotene in patients with acute promyelocytic leukemia relapsing after treatment with all-trans retinoic acid and arsenic trioxide. Br J Hematol 171: 4.

94. Wang JX, Mi YC, Jiang B, Chen XC, Ji CY, et al. (2015) Tamibarotene compared to all-trans retinoic acid (ATRA) as add-on to arsenic trioxide (ATO) in subjects with relapsed acute promyelocytic leukemia (APL). Blood 126: 220.
95. Kojima M, Ogiya D, Ichiki A, Hara R, Amaki J (2016) Refractory acute promyelocytic leukemia successfully treated with combination therapy of arsenic trioxide and tamibarotene. Leukemia Research Reports 5: 11-13.

96. Asou N (2017) Retinoic acid, all-trans retinoic acid (ATRA) and Tamibarotene Chemotherapy for leukemia. Springer pp. 183-211.

Copyright: $\mathbb{C} 2018$ Zhu G. This is an open-access article distributed under the terms of the Creative Commons Attribution License, which permits unrestricted use, distribution, and reproduction in any medium, provided the original author and source are credited. 